Original Article

\title{
Epidemiology of Urinary Tract Infections in The Preschool Children in Zagazig University Hospital
}

\author{
Ezzat K. Amin, Ali M. Abo zaid, Abd El Rahman I.Kotb.
}

Department of, Pediatrics Faculty of Medicine, Zagazig University

\begin{abstract}
Background

Urinary Tract Infection is one of the most common bacterial infections among children.. It is noted that the risk of renal damage from Urinary Tract Infection is greatest in children younger than 5 years, thus early diagnosis and prompt treatment are important.
\end{abstract}

\section{Aim of Work}

The aim of this study was to assess the prevalence of urinary tract infection in children attending Pediatric outpatient clinic in Zagazig University Children's Hospital. Also it aimed to determine, related risk factors, isolate the organisms that cause Urinary Tract Infection in children and antibiotics susceptibility patterns

\section{Patients and Methods}

This Cross sectional descriptive study, was conducted on 600 children, (377 males and 223 females) from 2 to 7 years old attending to pediatric outpatient clinic Zagazig University Children's Hospital, All patient groups were subjected to full medical history, physical examination, Dipstick analysis by using both nitrite and leukocyte esterase detector, Microscopic examinations and urine culture for positive cases.

\section{Results}

The prevalence of Urinary Tract Infection among children included in the current study was (7\%). Leukocyte esterase positive were $56(9.3 \%)$, Nitrite positive were 47 (7.8\%) and both Leukocyte esterase and Nitrite positive were $17(2.8 \%)$.

\section{Conclusion}

The prevalence of Urinary Tract Infection in our study was 7\%. Escherichia Coli was the most common organism. Cefotaxime and Amikacin were the most common antibiotic sensitive to the isolates.

\section{Keywords}

Urinary tract infections, urine culture, nitrite and leukocyte esterase.

\section{Correspondence}

\section{Ezzat Kamel Amin}

Faculty of Medicine, Zagazig University, Sheigk Abu Hamad st. AbuHamad City, Sharqia Governorate, Egypt.

Email: dr_ezat_kamel@yahoo.com

\author{
geget : The Journal of the Egyptian Society of Pediatric Nephrology and Transplantation (ESPNT) \\ geget https://geget.journals.ekb.eg/ \\ Published by ESPNT http://espnt.net/ \\ Cohosted by Egyptian Knowledge Bank https://www.ekb.eg
}




\section{Introduction}

Urinary Tract Infection (UTI) is one of the most common bacterial infections among children [1]. Approximately, $2 \%$ of boys and $8 \%$ of girls are affected at the age of 7 years old [2]. Urinary tract infection in children is significant source of morbidity. It is noted that the risk of renal damage from UTI is greatest in children younger than 5 years, thus early diagnosis and prompt treatment are important [3]. Factors predispose children to UTI include congenital and functional Abnormalities. Accurate diagnosis is important to ensure adequate therapy and proper follow up is essential to prevent future complication [4]. Seeking laboratory confirmation of diagnosis requires the initial stage of collecting an uncontaminated urine sample and this is a challenge in infants and children who are not toilet trained [5]. Use of dipstick test decrease

\section{Patients and Method}

This Cross sectional descriptive study was conducted from February to July 2017 at outpatient clinic, at Zagazig University Children's Hospital. This study was carried out on 600 children, ( 377 males and 223 females) from 2 to 7 years old attending to pediatric outpatient clinic.

\section{Inclusion criteria}

Children were selected in random way considered legible for entry into the study as they met the following criteria:

- Age from 2 - 7 years old.

- Live in Sharkia Governorate and Attended to Pediatric outpatient clinic in Zagazig University Children's Hospital.

\section{Exclusion criteria}

- Age below 2 years old and above 7 years old.

- A child with a catheter associated UTI.

- A child diagnosed with congenital anomalies.

- Previous surgery of the genitourinary tract (except circumcision in male children).

- A child with history of chronic renal diseases and Presence of an immunocompromising condition. (e.g., HIV, malignancy, use of chronic corticosteroids or other immunosuppressive agents).

Explanation of the purpose of the study to the parents was done.

Methods All the children included in the study were subjected to the following:

1- Detailed history taking with special focus on the presence of urological manifestations (dysuria, loin pain, frequency, supra-pubic pain, change of the colour of urine, enuresis), treatment with certain drugs for long

\section{Results}

The prevalence of UTI among children included in the current study was (7\%) (Table 1 and Figure 1). Results of the current study show that Children with dipstick LE positive were $56(9.3 \%)$, Nitrite positive were $47(7.8 \%)$ and both LE and Nitrite positive were 17 (2.8\%). LE sensitivity is $(73.8 \%)$, specificity $(95.5 \%)$, negative predictive value $(98 \%)$, positive predictive value $(55.4 \%)$ and overall accuracy (94\%). Nitrite sensitivity is (66.7\%), patient's time and money, and may also help in earlier initiation of treatment. Although culture is a gold standard for diagnosis of UTI, it has some disadvantages. Urine culture taken at least 48 hours, well equipped laboratory and trained staff give a reliable result. Whereas dipstick tests have the advantage of being rapid and easy to carry out and can be performed in small laboratories by laboratory technicians [6]. Using prevalence rates as an estimate of the prior probability of disease is the first step in evidence-based practice. In children with low pretest probability of disease, routine diagnostic testing is not necessary. In contrast, in children with high pretest probability of disease, routine diagnostic testing would be appropriate [7].

period, past history of renal diseases or family history of renal diseases. Full

2- Clinical examination: With special focus on weight, height and abdominal examination for renal mass or fullness of renal angles.

3- Urine samples from children :Collected by midstream clean catch urine sample in sterile cups then then Complete urine analysis were done on this urine specimens by Microscopic examinations where centrifuged urine was examined microscopically for pus cells, WBCs and RBCs morphology. Dipsticks analysis was done by using both esterase and nitrate detectors.

4- Urine culture was done for positive cases.

\section{Statistical Analysis}

All data were collected, tabulated and statistically analyzed using SPSS 20.0 for windows (SPSS Inc., Chicago, IL, USA) and MedCalc 13 for windows (MedCalc Software bvba, Ostend, Belgium). Quantitative data were expressed as the mean \pm SD \& range, and qualitative data were expressed as absolute frequencies (number) \& relative frequencies (percentage). Continuous data were checked for normality by using Shapiro Walk test. Percent of categorical variables were compared using Chi-square test or Fisher's exact test when appropriate. Validity of multistick test in diagnosis of UTI was calculated using diagnostic performance depend on sample $2 \times 2$ contingency tables generation using urine culture as gold standard reference. Sensitivity, specificity, positive predictive value, negative predictive value, accuracy were calculated. All tests were two sided. $\mathrm{P}<0.05$ was considered statistically significant $(\mathrm{S}), \mathrm{p}<0.001$ was considered highly statistically significant (HS), and $\mathrm{p} \geq 0.05$ was considered non statistically significant (NS).

specificity (96.6\%), negative predictive value $(97.5 \%)$, positive predictive value $(59.6 \%)$ and overall accuracy $(94.5 \%)$.

Both Leukocyte esterase (LE) and nitrite sensitivity is $(40.5 \%)$, specificity $(100 \%)$, negative predictive value $(95.7 \%)$, positive predictive value $(100 \%)$ and overall accuracy $(95.8 \%)$. Risk factors of UTI in children in our study were uncircumcised males, 
prolonged use of antibiotics, family history of UTI, recurrent UTI, enuresis, and constipation. Escherichia Coli was the most common organism that cause UTI in children in our study .Cefotaxime and Amikacin were the most common antibiotic sensitive to the isolate.

Table 1 : Prevalence of UTI

\begin{tabular}{|c|c|c|}
\hline \multirow{2}{*}{ UTI } & \multicolumn{2}{|c|}{$\begin{array}{c}\text { All studied sample } \\
\text { (N=600) }\end{array}$} \\
\cline { 2 - 4 } & No. & $7 \%$ \\
\hline Positive & 42 & $93 \%$ \\
\hline Negative & 558 & $7 \%$ \\
\hline
\end{tabular}

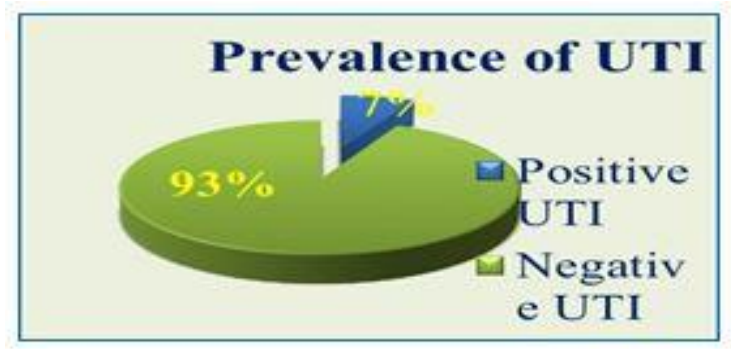

Figure 1: Prevalence of UTI

\section{Discussion}

This study was an attempt to determine the prevalence of urinary tract infection, microbial pathogens implicated in urinary tract infection and their antibiotic susceptibility patterns in children attended to pediatric outpatient clinic at Zagazig University Children's Hospital. The total number of children included in this study was 600 children from age 2-7 years, 377 child $(62.8 \%)$ were males and 223 child $(37.2 \%)$ were females, 179 child $(29.8 \%)$ from urban areas and 421 child $(70.2 \%)$ from rural areas.

This study shows that the prevalence of urinary tract infection in children attended to pediatric outpatient clinic at Zagazig University Children's Hospital was 42 (7\%) child from 600 child included in this study. Similar to our results [8]. In Giza governorate in Egypt found that the Prevalence of UTI of 1000 apparently healthy school going children, 552 boys $(55.2 \%)$ and 448 girls (44.8\%), were enrolled in this cross sectional prevalence survey was $6 \%$ among school aged children.

In the study of Durmišiević, et al. [9], in Bosnia and found that UTI was present $352(6.5 \%)$ children, among those $114(32.4 \%)$ were boys and $238(67.6 \%)$ girls. In the study of Shaikh, N., et al. [7], the overall prevalence of UTI in older children ( $\leq 19$ years) with or without fever is reported to be $7.8 \%$. In the study of Akujobi, C., et al. [10], in Nnewi in Nigeria the prevalence of significant bacteriuria among pediatric patients attending Nnamdi Azikiwe University Teaching Hospital is $8 \%$, this study showed that there was high incidence of UTI among the female gender $(66.67 \%)$ than the male gender $(33.33 \%)$.

Another study in Menoufiya Governorate in Egypt in study of Abdelhamid et al, [11], Out of 500 children 314
$(62.8 \%)$ males and $186(37.2 \%)$ females, the prevalence of UTIs among children aged 3-12 years attending to Pediatric Outpatient Clinic in Menoufia University Hospital was (4.8\%).On the contrary to our study, Mahmoud,. et al. 2016 [12], in Sohag governorate in Egypt where the prevalence of UTIs among primary school children from five primary schools in different parts of the Sohag Governorate, was $0.6 \%$ for the initial urinary screening and $0.5 \%$ for the second screening.

Results of the current study shows that there was statistically significant difference between males and females in relation to prevalence of UTI, where prevalence of UTI in females was $54.8 \%$ (23 females from 42 patients with UTI) while prevalence of UTI in males was $45.2 \%$ (19 males from 42 patients with UTI ) $(\mathrm{P}=0.014)$, the highest number of cases $(31 \%)$ was found among females in age group 5-7 years then among males $(28.6 \%)$ in age group 24 years. Similar to our results, Mohammed., et al.[8] higher prevalence of UTI occurred in girls $(11.4 \%)$ than boys $(1.6 \%)$, with statistically significant difference between males and females in relation to prevalence of UTI. In the study of El-Shafie. et al [13], there was statistically significant difference between males and females in relation prevalence of UTI among the screened children, (Table 2). 
Table 2: Relationship between sex and prevalence of UTI in the studied cases.

\begin{tabular}{|c|c|c|c|c|c|c|c|c|}
\hline \multirow{3}{*}{ Sex } & \multicolumn{4}{|c|}{ UTI } & & & \multirow{3}{*}{ Test } & \multirow{3}{*}{$\begin{array}{l}\text { p-value } \\
\text { (Sig.) }\end{array}$} \\
\hline & \multicolumn{2}{|c|}{ Negative UTI } & \multicolumn{2}{|c|}{ Positive UTI } & \multicolumn{2}{|c|}{ Total } & & \\
\hline & No. & $\%$ & No. & $\%$ & No. & $\%$ & & \\
\hline Female & 200 & $35.8 \%$ & 23 & $54.8 \%$ & 223 & $37.2 \%$ & & \\
\hline Male & 358 & $64.2 \%$ & 19 & $45.2 \%$ & 377 & $62.8 \%$ & & $\begin{array}{r}0.014 \\
(S)\end{array}$ \\
\hline Total & 558 & $93 \%$ & 42 & $7 \%$ & 600 & $100 \%$ & 5.987 & \\
\hline
\end{tabular}

\$ Chi-square test. $\quad \mathbf{P}<\mathbf{0 . 0 5}$ is significant.

This table shows that there was statistically significant difference between males and females in relation to prevalence of UTI, where the prevalence of UTI in female $54.8 \%$

(23 female from 42 one has UTI) while the prevalence of UTI in male $45.2 \%$ (19 male from 42 one has UTI) $(\mathrm{P}=0.014)$

Results of the current study show statistically significant difference between risk factors as uncircumcised males, family history of UTI, recurrent UTI,

In the study of Sawalha [14], nocturnal enuresis was highly significant to prevalence of UTI $(P=0.035)$ .In the study of Safarinejad [15], the overall prevalence of enuresis was $6.8 \%$, the prevalence of urinary tract pathology was $2.9 \%$ among enuretics which indicates high association between UTI and nocturnal enuresis. In the study of Afridi, et al. [16], circumcised males appear to be at lower risk for developing UTI perhaps because of low perurethral and urethral bacterial inoculums, this may be due to the small number of males included in the study. In the study of Isa, M., et al [17] reported that there were enuresis, and constipation in relation to prevalence of UTI, and statistical significant higher difference between prolonged use of antibiotic in relation to prevalence of UTI. statistically significant difference between bed wetting and UTI. However, the association between UTI and previous history of UTI was insignificant $(P>0.05)$ in the study of Hossain., et al [18], showed that female sex $(\mathrm{P}<0.05)$, constipation $(\mathrm{P}<0.001)$, not taking anthelmintic $(\mathrm{P}<0.001)$, lack of toilet training $(\mathrm{P}<0.001)$ and inadequate water intake $(\mathrm{P}<0.01)$ were significant risk factors for urinary tract infection in children, (Table 3 ).

Table 3: Risk factors for UTI in the studied population.

\begin{tabular}{|c|c|c|c|c|c|c|c|c|c|}
\hline \multirow{3}{*}{\multicolumn{2}{|c|}{ Risk factors }} & \multirow{2}{*}{\multicolumn{2}{|c|}{$\begin{array}{c}\text { Total } \\
(\mathbf{N}=600)\end{array}$}} & \multicolumn{4}{|c|}{ UTI } & \multirow{3}{*}{ Test } & \multirow{3}{*}{$\begin{array}{l}\text { P-value } \\
\text { (Sig.) }\end{array}$} \\
\hline & & & & \multicolumn{2}{|c|}{$\begin{array}{c}\text { Negative UTI } \\
(\mathrm{N}=558)\end{array}$} & \multicolumn{2}{|c|}{$\begin{array}{c}\text { Positive UTI } \\
(\mathrm{N}=42)\end{array}$} & & \\
\hline & & No. & $\%$ & No. & $\%$ & No. & $\%$ & & \\
\hline \multirow{2}{*}{ Uncircumcised Males } & Absent & 594 & $99 \%$ & 555 & $93.4 \%$ & 39 & $6.6 \%$ & \multirow{2}{*}{17.214} & \multirow{2}{*}{$\begin{array}{l}0.006 \\
(\mathrm{~S})\end{array}$} \\
\hline & Present & 6 & $1 \%$ & 3 & $50 \%$ & 3 & $50 \%$ & & \\
\hline \multirow{2}{*}{ Prolonged use of antibiotics } & Absent & 579 & $96.5 \%$ & 547 & $94.5 \%$ & 32 & $5.5 \%$ & \multirow{2}{*}{55.153} & \multirow{2}{*}{$\begin{array}{c}<0.001 \\
(\mathrm{HS})\end{array}$} \\
\hline & Present & 21 & $3.5 \%$ & 11 & $52.4 \%$ & 10 & $47.6 \%$ & & \\
\hline \multirow{2}{*}{ Family history of UTI } & Absent & 595 & $99.2 \%$ & 555 & $93.3 \%$ & 40 & $6.7 \%$ & \multirow{2}{*}{8.434} & \multirow{2}{*}{$\begin{array}{c}0.042 \\
(\mathrm{~S})\end{array}$} \\
\hline & Present & 5 & $0.8 \%$ & 3 & $60 \%$ & 2 & $40 \%$ & & \\
\hline \multirow{2}{*}{ Recurrent UTI } & Absent & 586 & $97.7 \%$ & 549 & $93.7 \%$ & 37 & $6.3 \%$ & \multirow{2}{*}{18.155} & \multirow{2}{*}{$\begin{array}{l}0.002 \\
(\mathrm{~S})\end{array}$} \\
\hline & Present & 14 & $2.3 \%$ & 9 & $64.3 \%$ & 5 & $35.7 \%$ & & \\
\hline \multirow{2}{*}{ Enuresis } & Absent & 554 & $92.3 \%$ & 521 & $94 \%$ & 33 & $6 \%$ & \multirow{2}{*}{12.083} & \multirow{2}{*}{$\begin{array}{l}0.003 \\
(\mathrm{~S})\end{array}$} \\
\hline & Present & 46 & $7.7 \%$ & 37 & $80.4 \%$ & 9 & $19.6 \%$ & & \\
\hline \multirow{2}{*}{ Constipation } & Absent & 562 & $93.7 \%$ & 526 & $93.6 \%$ & 36 & $6.4 \%$ & \multirow{2}{*}{4.814} & \multirow{2}{*}{$\begin{array}{l}0.041 \\
(\mathrm{~S})\end{array}$} \\
\hline & Present & 38 & $6.3 \%$ & 32 & $84.2 \%$ & 6 & $15.8 \%$ & & \\
\hline
\end{tabular}

$\begin{array}{lll}\ddagger \text { Chi-square test. } \quad \mathrm{P}<0.05 \text { is significant. } & \text { Sig: significance. }\end{array}$

Results of the current study show that Children with dipstick LE positive were $56(9.3 \%)$, nitrite positive were $47(7.8 \%)$ and both LE \& Nitrite positive were 17 (2.8\%). LE sensitivity is $(73.8 \%)$, specificity $(95.5 \%)$, negative predictive value $(98 \%)$, positive predictive value $(55.4 \%)$ and overall accuracy (94\%). Nitrite sensitivity is $(66.7 \%)$, specificity $(96.6 \%)$, negative predictive value $(97.5 \%)$, positive predictive value $(59.6 \%)$ and overall accuracy $(94.5 \%)$. Both Leukocyte esterase (LE) and nitrite sensitivity is $(40.5 \%)$, specificity $(100 \%)$, negative predictive value $(95.7 \%)$, positive predictive value $(100 \%)$ and overall accuracy (95.8\%). Similar to our results, ElShafie, et al. [13], found that the sensitivity of the dipstick was $82.6 \%$, specificity was $98.9 \%$, positive predictive value was $79.1 \%$ and negative predictive value was 99.1 $\%$. So dipstick is a good negative test rather being a good positive test for detection of UTI. However, in the study of Najeeb., et al. [6], found that Combined sensitivity of LE and Nitrite was $75.74 \%$ while specificity was $68.90 \%$. He concluded that urine dipstick test may be considered for rapid urinalysis to diagnose UTI, (Table 4 and Figure 2). 
In the study of Laosu-angkoon [19], found that the sensitivity of leukocyte esterase test were $63.6 \%$ while the combined leukocyte esterase and nitrite test were $66.7 \%$. He concluded that the dipstick test can be used as a diagnostic tool in detecting UTI cases in ER to prevent potential sequel like hypertension and renal scarring.

The study of Taneja, N., et al. [20]), found that combined sensitivity of LE and Nitrite was $79.6 \%$, while sensitivity and specificity of LE were $73.5 \%, 58.5 \%$ respectively and for Nitrite were $57.1 \%, 78.7 \%$ respectively. He concluded that for faster diagnosis of UTI, dipstick tests for leukocyte esterase and nitrite test should be added in routine laboratory practices.

The study of Abdelhamid et al, [11], found that LE sensitivity is $(85.8 \%)$, specificity $(54.1 \%)$, positive predictive value $(45.9 \%)$ and negative predictive value $(91.2 \%)$. Nitrite sensitivity is $(79.3 \%)$, specificity $(66.3 \%)$, positive predictive value $(73.9 \%)$ and negative predictive value (88.9\%). Leukocyte esterase (LE) and nitrite sensitivity is $(71.2 \%)$, specificity $(100.00 \%)$, positive predictive value $(100 \%)$ and negative predictive value $(79.4 \%)$.

Table 4: Specificity and sensitivity of multi-stick method in relation to culture methods regarding UTI.

\begin{tabular}{|c|c|c|c|c|c|}
\hline & Sensitivity & Specificity & NPV & PPV & $\begin{array}{c}\text { Overall } \\
\text { accuracy }\end{array}$ \\
\hline LE & $73.8 \%$ & $95.5 \%$ & $98 \%$ & $55.4 \%$ & $94 \%$ \\
\hline Nitrite & $66.7 \%$ & $96.6 \%$ & $97.5 \%$ & $59.6 \%$ & $94.5 \%$ \\
\hline LE \& Nitrite & $40.5 \%$ & $100 \%$ & $95.7 \%$ & $100 \%$ & $95.8 \%$ \\
\hline
\end{tabular}

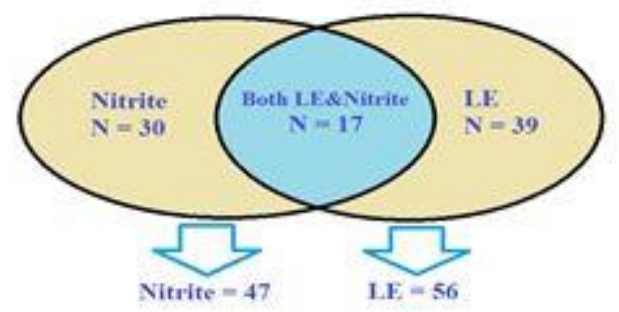

Figure 2: Results of dipstick tests in studied sample.

Results of the current study show that according to culture, the most common infecting organism was E.coli that represent $(76.2 \%)$ followed by Klebsiella pneumonia $(9.5 \%)$, Enterococcus Faecalis $(7.1 \%)$, Proteus mirabilis $(4.8 \%)$ and coagulase -ve staphylococci in $(2.4 \%)$, (Table 5 and Figure 2)

Similar to our results, Lehrasab, et al. [21], the most common organisms responsible for urinary tract infection (UTI) found were E Coli (47.6\%) followed by Klebsiella $(23.2 \%)$ and Proteus (10\%), Staphylococcus was found in $(7.3 \%)$ and Enterobacter in $(7.3 \%)$ children, and rest $(4.6 \%)$ were effected with other organisms.

The study of Abdelhamid et al, [11], shows that according to culture, the infecting organism was E.coli in $(62.5 \%)$, Klebsiella in (12.5\%), Enterococcus Faecalis in
(16.7\%) and coagulase -ve staphylococci in (8.3\%). In the study of Dada, E. and C. Aruwa [22], the predominant bacterial isolate was E. coli $(56.8 \%)$, followed by S. aureus (18.9\%), Klebsiella spp. (16.2\%) and P. aeruginosa $(8.1 \%)$.

In the study of Afridi, et al., [16], the most common urinary pathogens isolated were E.coli (63\%), Klebsiella pneumoniae $(8 \%)$ and Proteus mirabilis $(8 \%)$. Other pathogens included Pseudomonas auerogenosa (7\%), Staphylococcus aureus and Citrobacter 5\% each and Enterobacter and Coliform $2 \%$ each. In study of El-Shafie, et al [13] the most common organism found in UTI cases culture was E coli (62 \%), Enterococcus faecalis $(17.3 \%)$, Klebsiella pneumonia $(10.3 \%)$, and Coagulase negative staphylococci(10.4\%).

Table 5: Infecting organisms in children with culture.

\begin{tabular}{|c|c|c|}
\hline \multirow{2}{*}{ Infecting organisms } & \multicolumn{2}{|c|}{ Positive Culture $(\mathbf{N}=\mathbf{4 2})$} \\
\cline { 2 - 3 } & No. & \% \\
\hline E. coli & 32 & $76.2 \%$ \\
\hline Klebsiella pneumonia & 4 & $9.5 \%$ \\
\hline Enterococcus fecalis & 3 & $7.1 \%$ \\
\hline Proteus mirabilis & 2 & $4.8 \%$ \\
\hline Coagulase -ve staphylococci & 1 & $2.4 \%$ \\
\hline
\end{tabular}




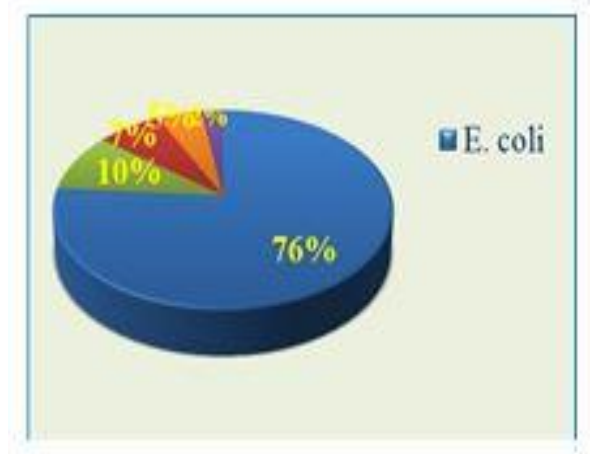

Figure 3: Infecting organisms in children with culture.

Results of the current study show that according to antibiotic sensitivity, 12 (28.6\%) patients were sensitive to Cefotaxime, $11(26.2 \%)$ were sensitive to Amikacin, 6 $(14.3 \%)$ were sensitive to Amoxicillin and Clavulanic acid, $3(7.1 \%)$ were sensitive to Ceftriaxone, $3(7.1 \%)$ were sensitive to Co Trimoxazole, $3(7.1 \%)$ were sensitive to Imipenem, $2(4.8 \%)$ were sensitive to Ciprofloxacin and 2 $(4.8 \%)$ were sensitive to Nitrofurantoin. Similar to our study of Abdelhamid et al, [11], shows that according to antibiotic sensitivity, $(62.5 \%)$ patients were sensitive to cefotaxime, $(25 \%)$ were sensitive to Amikacine and $(12.5 \%)$ were sensitive to Amoxicillin and clavulanic acid, (Table 6 and Figure4).

In the study of $\mathrm{Wu}, \mathrm{T} .-\mathrm{H}$., et al [23], the most common uropathogen in primary and recurrent UTIs is E. coli and cefazolin is the drug of choice for treating such infections in children. In study of Afridi, et al.,[16], Sensitivity of different urinary isolated to Amikacin was highest (82\%) followed by meropenem (75\%), tazocin (61\%) and Sulzone $(58 \%)$. In the study of Sharma, A., et al. [24], most of the organisms were highly sensitive to Nitrofurantoin and Amikacin. Sensitivity to quinolones and third generation Cephalosporins varied according to the organism. E. coli was $100 \%$ sensitive to Nitrofurantoin. E. coli was sensitive to ofloxacin, cefotaxim and amikacin in $94.4 \%, 94.7 \%$ and $94.7 \%$, respectively. E. coli was resistant to ampicillin in $91.6 \%$, cotrimoxazole in $66.6 \%$ and nalidixic acid in 63.6 $\%$. Our study is small sample size, not cover large population but may help in meta analysis studies.

Table 6: Number and percentage distribution of antibiotic sensitivity.

\begin{tabular}{|c|c|c|}
\hline \multirow{2}{*}{ Antibiotic sensitivity } & \multicolumn{2}{|c|}{ Positive Culture (N=42) } \\
\cline { 2 - 4 } Cefotaxime & No. & $28.6 \%$ \\
\hline Amikacin & 12 & $26.2 \%$ \\
\hline Amoxicillin/Clavulanic & 11 & $14.3 \%$ \\
\hline Ceftriaxone & 6 & $7.1 \%$ \\
\hline Co Trimoxazole & 3 & $7.1 \%$ \\
\hline Imipenem & 3 & $7.1 \%$ \\
\hline Ciprofloxacin & 3 & $4.8 \%$ \\
\hline
\end{tabular}




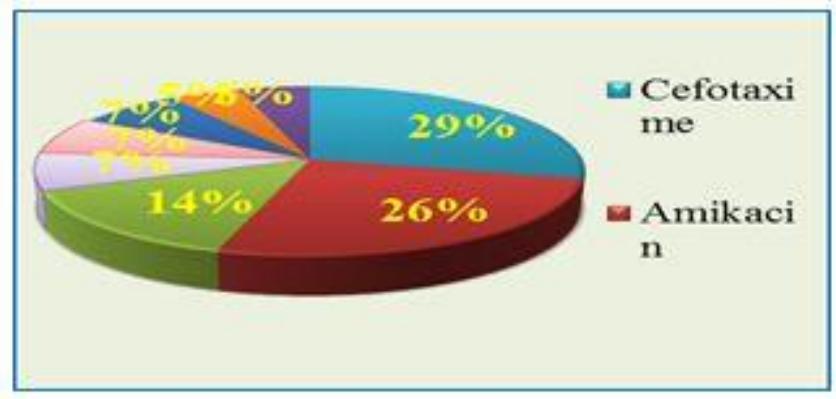

Figure 4: Percentage of distribution of antibiotic sensitivity.

\section{Conclusion and Recommendations}

We conclude that the prevalence of UTI in our study was $7 \%$.Risk factors of UTI in children in our study were uncircumcised males, prolonged use of antibiotics, and family history of UTI, recurrent UTI, enuresis, and constipation. Escherichia Coli was the most common organism that cause UTI in children in our study. Cefotaxime and Amikacin were the most common antibiotic sensitive to the isolates, Further studies must be done in large population overall Egypt to help in determination of local causative organisms and preventive measures to decrease the prevalence of UTI by knowing the risk factors and preventing it .

\section{References}

1- Mi Mi Oh, Jun Cheon, Seok Ho Kang. "Predictive factors for acute renal cortical scintigraphic lesion and ultimate scar formation in children with first febrile urinary tract infection." The Journal of urology.2010.

2- Craig JC1, Simpson JM, Williams GJ, Lowe A, Reynolds GJ, McTaggart SJ. " Antibiotic prophylaxis and recurrent urinary tract infection in children."

3-N Engl J Med, 2010, (361): 18.

4- Saeed, C.H.; AL-Otraqchi, K.I. and Mansoor, B.I.Y. "Prevalence of Urinary Tract infections and antibiotics susceptibility pattern among infants and young children in Erbil city. Zanco J. Med. Sci, 2015, 19 (1): 915-922.

5- White, Brett. "Diagnosis and treatment of urinary tract infections in children." American family physician 2011, Feb 15; 83 (4):409-415.

6- Colgan, R. and M. Williams. "Diagnosis and treatment of acute uncomplicated cystitis."

American family physician 2011, 84 (7): 771.

7- Najeeb, Sara Munir, Tehmina Rehman, Sabahat Hafiz, "Comparison of urine dipstick test with conventional urine culture in diagnosis of urinary tract infection."

J Coll Physicians Surg Pak. 2015, 25(2): 108-110.

8- Shaikh, Nader Morone, Natalia E Bost, James E Farrell, Max H. "Prevalence of urinary tract infection in childhood: a meta-analysis " The Pediatric infectious disease journal; 2007, 11(5):479-481.

9- Mohammed, Ahmed Abdelfattah, Magid Ibraheem, Ayman Younes, "A study of asymptomatic bacteriuria in Egyptian school-going children." African health sciences 2016, 16(1): 69-74. (4): 302-308.
10- Durmišiević.Serdarević, Jasminka ,Durmišević, Smajil Lelić, Melita Durmišević. "Urinary tract infections in preschool children."

Medicinski Glasnik. 2013, 10 (1):28-34.

11- Akujobi, CN Ezeanya, CC Emeka Okafor, KM Ebenebe, JC "A Study on Significant Bacteriuria among Children Attending the Out-Patient Clinic of a University Teaching Hospital, Nigeria." International Journal of Microbiology Research, 2013, 5(4): 448.

12- Waled Abd El Ghaffar Abdelhamid. "Prevalence of Urinary Tract Infection in Children Attending Pediatric Outpatient Clinic in Menoufia University Hospital. Thesis Form Submitted for fulfillment of M.Sc. degree in Pediatrics, 2016, 53:59.

13- Mahmoud A .Ramadan, El Masry Al-zahraa E Mohammad, Radwa S Yousef, Fouad. "Urinary Screening for Detection of Renal Abnormalities in Asymptomatic School Children, Sohag Governorate, Egypt."

BJMMR, 2016, 13 (6): 1-8.

14- El-Shafie, Ali M El-Nemr, Fathia M Bahbah, MB Shokry, Mohamed Attia, A. "The role of urine screening (in school children of Menoufiya Governorate) in early detection of renal disorders." Journal of American Science. 2014, 10: 143-150.

15- Sawalha, Raya Mohammad Hussein. P. prevalence of Urinary Tract Infection among Children of Primary Schools in Nablus, Thesis is submitted in partial Fulfillment of the Requirements for the degree of Master of Public Health Science, Faculty of Graduate Studies, An-Najah National University, 2009, 33-39.

16- Safarinejad, Mohammad. R."Prevalence of nocturnal enuresis, risk factors, associated familial factors and urinary pathology among school children in Iran." Journal of pediatric urology. 2007, 3(6): 443-452.

17- Afridi, Jahanzeb Khan Afridi, Mukhtiar Ahmad Karim, Rahida Munir, Arshia. "Causative organisms and their sensitivity pattern of urinary tract infection in children of a tertiary care hospital."

KJMS, 2014, 7(2): 291-294.

18- Isa, MA Ismail, HY Allamin, IA Shettima, A Mustapha, A. "Prevalence of Urinary Tract Infection among Primary School Children in Maiduguri, Borno State, Nigeria."

International Journal of Environment, 2013, 2 (1): 9-15.

19- Hossain, MA, Akter, R, Mannan KA, Ahmed MS, Deb KP, et al." Risk Factors of Febrile Urinary Tract Infection in Children." Urology \& Nephrology Open Access Journal; 2015, 2 (5):138-141. 
20- Laosu-angkoon, Sirirat,. "The sensitivity and specificity of a urine leukocyte esterase dipstick test for the diagnosis of urinary tract infection in the outpatient clinic of Rajavithi Hospital."

Journal of the Medical Association of Thailand= Chotmaihet thangphaet, 2013, 96(7): 849-853.

21- Taneja, N Chatterjee, SS Singh, M Sivapriya, S Sharma, M Sharma, SK. "Validity of quantitative unspun urine microscopy, dipstick test leucocyte esterase and nitrite tests in rapidly diagnosing urinary tract infections." J Assoc Physicians India. 2010, 58: 485-487.

22- Lehrasab, Waseem Aziz, Tahir Ahmed, Naheem Ahmed, Imtiaz."Causative Organisms of Urinary Tract Infection and their Sensitivity Pattern in Children."

Ann. Pak.Inst. Med.Sci, 2016, 12 (4):181-5.
23- Dada, EO Aruwa, CE. "Asymptomatic Bacteriuria Prevalence among Primary School Children in the Federal University of Technology, Akure (Futa), Ondo State, Nigeria."Journal of Applied Life Sciences International, 2016, 4(4): 1-8.

24- Wu, Tsung-Hua Huang, Fang-Liang Fu, Lin-Shien Chou, Chia-Man Chien, Ya-Li Huang, Chung-Ming Lin, Chin-Fu Chen, Po-Yen. "Treatment of recurrent complicated urinary tract infections in children with vesicoureteral reflux." Journal of Microbiology, Immunology and Infection, 2016, 49(5): 717-722.

25- Sharma, A Shrestha, S Upadhyay, S Rijal, P.. "Clinical and bacteriological profile of urinary tract infection in children at Nepal Medical College Teaching Hospital." Nepal Med Coll Journal, 2011, 13(1): 24-26.

\section{Declaration}

\section{Ethics approval and consent to participate}

This study protocol and the consents were approved and deemed sufficient by Ethical Committee of Pediatric Department, Faculty of Medicine, Zagazig University. And informed written consent was obtained in every case from their legal guardians.

\section{Funding}

The authors declare that they didn't receive any financial support from agencies or others.

\section{Conflict of interest}

No

\section{Acknowledgements}

We would like to thank all patients and their family members for their valuable contributions to the study. 